\title{
Engaging Future Generations of ACM Leaders
}

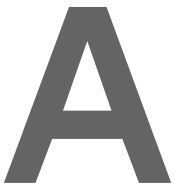

TTRACTING YOUNGER, NEXTGENERATION members is a challenge faced by professional societies worldwide. ACM recognized early on that as a volunteer-driven organization, our future health depends not only on appealing to a diverse membership, but also on creating a pipeline of future leaders. We are in the process of developing new strategies to motivate recent graduates to get involved with ACM. In the meantime, I'd like to tell you about two programs that have already struck a chord with early-career professionals. Both were designed to offer young technologists and researchers the opportunity to network with peers from around the globe and to hone their leadership skills through personal interactions with more experienced leaders.

The ACM Future of Computing Academy (FCA) was created in 2016 to help shape the future of our organization. It brings together talented young professionals from a broad range of computing subdisciplines and institutions-academia, research labs, established companies, and startups-who want to perform valuable services for the community while expanding their professional networks and leadership experience. FCA members identify and implement pilot projects that address challenging issues facing our organization and the computing field in general. They work closely with ACM leadership, bringing new ideas/perspectives and helping to integrate successful pilots into ACM's institutional practices.

FCA members are selected biennially through a highly competitive process similar to those used for ACM awards. This summer's recruitment attracted hundreds of applications from around the globe, resulting in 36 new members.

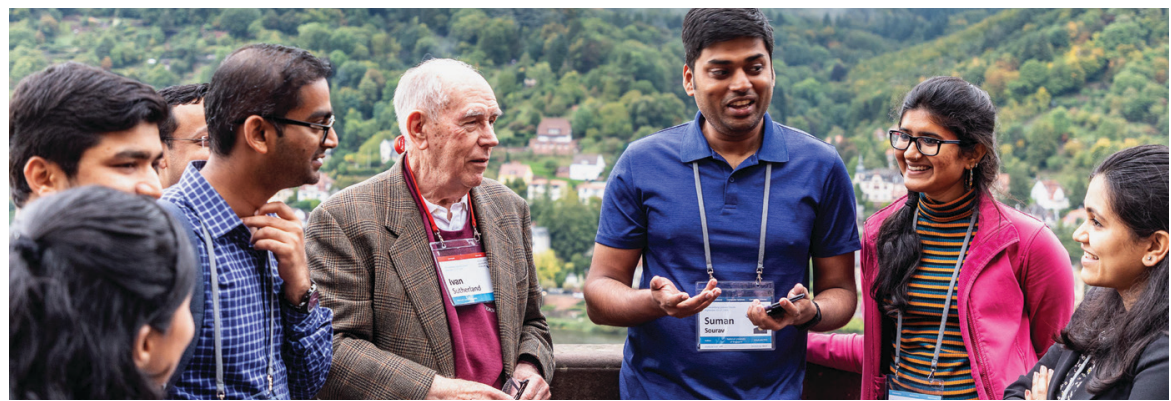

1988 ACM A.M. Turing Award recipient Ivan Sutherland (fourth from right) visits with young researchers at the Heidelberg Laureate Forum last September.

The expanded membership represents 17 countries on all six inhabited continents. It is an extremely diverse group, in terms not just of gender and geography but also type of organization and disciplinary interests. Learn more about the FCA and its activities at https://www. acm.org/fca.

The Heidelberg Laureate Forum (HLF) is an annual event where young researchers in computing or mathematics share the scientific stage with world leaders who have been recognized with the ACM A.M. Turing Award, ACM Prize in Computing, Abel Prize, Fields Medal, or Nevanlinna Prize. ACM partnered with other leading scientific organizations to launch HLF in 2013. The event provides a unique opportunity for young researchers to interact with some of our industry's greatest innovators. HLF's week-long program is a carefully crafted mix of research updates, panels on controversial issues in the computing and math fields, group discussions, and informal social activities.

Approximately 200 young researchers are accepted each year for HLF, which takes place in Heidelberg (Germany) at the end of September. As with FCA, the application process is extremely competitive. Participants include undergraduates, postgraduate students, and post- docs in approximately equal proportion. The 2019 event hosted 202 young researchers from 60 nations, 120 male and 82 female, many of them with interdisciplinary backgrounds. I strongly encourage young researchers to apply for next year's event. As you read this, the application portal has opened for the $8^{\text {th }}$ Annual HLF, slated for September 2020 (https://application.heidelberg-laureateforum.org/; the deadline is Feb. 14, 2020).

As a professional society, ACM leadership recognizes that the key to our long-term sustainability is to engage the ideas, energy, and enthusiasm of new generations. We must create opportunities for young professionals to grow their careers and give back to their communities through ACM. The ACM Future of Computing Academy and Heidelberg Laureate Forum are two examples where we have already seen success. But the journey has just begun. I encourage each of you to join in and help us explore the future of ACM. Share your own ideas with us about how to serve our growing community in new and forwardthinking ways.

C

Cherri M. Pancake is President of ACM, professor emeritus of electrical engineering and computer science, and director of a research center at Oregon State University, Corvallis, OR, USA

Copyright held by author/owner. 